\title{
Magnetization Current Simulation of High-Temperature Bulk Superconductors Using the ANSYS Iterative Algorithm Method
}

\author{
Kai Zhang, Sebastian Hellmann, Marco Calvi, Thomas Schmidt, Lucas Brouwer
}

\begin{abstract}
The $A-V$ formulation based iterative algorithm method (IAM), developed at Paul Scherrer Institute, shows excellent performance in modelling the critical state magnetization-current in the ReBCO tape stacks after field-cooling (FC) magnetization. This paper upgrades the function of the IAM, making it realistic to take into account the widely used $E$-J power law, as well as to model the magnetization-current in bulk high-temperature superconductors (HTS) under a zero-field-cooling (ZFC) cycle, having both the magnetization and demagnetization stages. The simulation results obtained from the ANSYS-IAM are validated with the well-known $\boldsymbol{H}$-formulation method for both the critical state model and the flux creep model. The computation times from using the two different numerical methods are compared and discussed. The influence factors, including the iteration steps, the initial superconductor resistivity, the ramping time and the reservation coefficient, are studied in detail.
\end{abstract}

Index Terms - HTS modelling, $A-V$ formulation, magnetization, ANSYS, $\boldsymbol{H}$-formulation, APDL

\section{INTRODUCTION}

Research and development work on implementing self-developed numerical methods into the finite-element method (FEM) software ANSYS is ongoing at the Paul Scherrer Institute (PSI) for the purpose of modelling and optimizing the short-period and high-field staggered-array high-temperature superconductor (HTS) bulk undulators which are highly appreciated for the construction of compact free electron lasers (FELs) [1]-[3]. The idea of developing ANSYS comes from its powerful capability for mechanical or multiphysics-coupled simulations and its parametric design language (APDL) which allows the secondary development or the user-defined element [4]. Our ambitious goal is to develop adaptive numerical algorithms which could allow the electromagnetic modelling of HTS obeying the critical state model or the flux creep model (e.g., $\boldsymbol{E}$-J power law), but with

This work is supported by European Union's Horizon2020 research and innovation program under grant agreement No. 777431 and European Union's Horizon2020 research and innovation program under the Marie Sklodowska-Curie grant number No. 701647.

Kai Zhang, Sebastian Hellmann, Marco Calvi and Thomas Schmidt are all with Paul Scherrer Institute, Villigen, 5232, Switzerland (e-mail: kai.zhang@psi.ch; $\quad$ sebastian.hellmann@psi.ch; $\quad$ marco.calvi@psi.ch; thomas.schmidt@psi.ch)

Lucas Brouwer is with Lawrence Berkeley National Laboratory, Berkeley, 94720, USA (email: LNBrouwer@lbl.gov) faster or equivalent computation speed in comparison to the $\boldsymbol{A}-\boldsymbol{V}$ formulation [5], [6], the $\boldsymbol{T}-\boldsymbol{\Omega}$ formulation [7], the $\boldsymbol{T}-\boldsymbol{A}$ formulation [8], [9], the well-known $\boldsymbol{H}$-formulation [10]-[14], or the Minimum Electro-Magnetic Entropy Production (MEMEP) method implemented in other FEM or home-made software [15].

ANSYS was first explored to solve the AC loss of HTS by Gu et al. in 2005 and then by Farinon et al. in 2010 using a resistivity adaption algorithm [16]-[18]. This algorithm aims at finding a final resistivity matrix for all superconductor elements but it is lacking computational efficiency. Recently, a new backward computation method based on ANSYS 18.1 Academic was proposed by Zhang et al. in 2020 to accelerate modelling of the critical state magnetization-current in a staggered-array HTS bulk undulator after a field-cooling (FC) magnetization [19]. This new method shows significantly faster computation speed than other state-of-the-art numerical methods, including the earlier ANSYS iterative algorithm method (IAM) proposed by Zhang et al. in 2019 [20]. Nevertheless, the ANSYS-IAM is still of great interest for HTS modelling since it provides real-time magnetization results at any intermediate steps during the magnetization process.

In this paper, as a follow-up of the ANSYS-IAM, we have for the first time taken into account the $\boldsymbol{E}-\boldsymbol{J}$ power law and explored the possibility of modelling the zero-field-cooling (ZFC) cycle which has both the magnetization and demagnetization stages. The simulation results of a bulk HTS are validated with the well-known $\boldsymbol{H}$-formulation method. The parameters which can affect the numerical results are studied in detail for both the critical state mode and the flux creep model.

The superconductor resistivity obeying the critical state model is

$$
\rho=\left\{\begin{array}{llll}
0 & \text { if } & \boldsymbol{J} \mid & J_{\mathrm{c}}(B) \\
+\infty & \text { if } & \boldsymbol{J} \mid & J_{\mathrm{c}}(B)
\end{array}\right.
$$

where $J_{\mathrm{c}}(B)$ refers to the magnetic-field-dependent critical current density and $|\boldsymbol{J}|$ refers to the absolute value of the current density.

The superconductor resistivity obeying the $\boldsymbol{E}-\boldsymbol{J}$ power law (flux creep model) is

$$
\rho=\frac{E_{\mathrm{c}}}{J_{\mathrm{c}}(B)} \cdot\left(\frac{|\boldsymbol{J}|}{J_{\mathrm{c}}(B)}\right)^{n-1}
$$



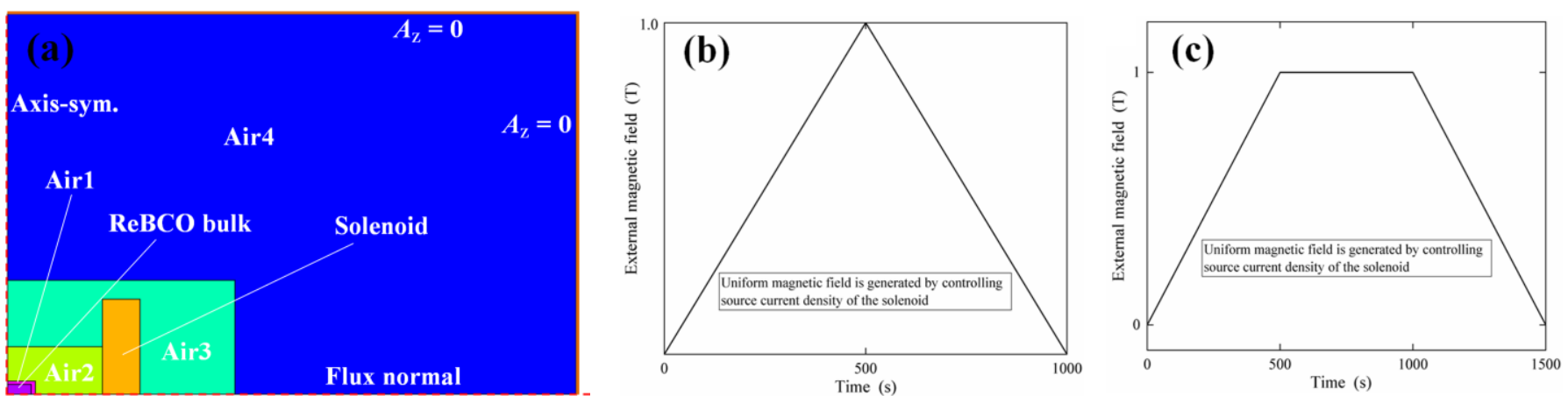

Fig. 1. (a) 2D axis-symmetric half FEA model; (b) ZFC magnetization cycle for critical state modelling; (c) ZFC magnetization cycle for flux creep state modelling.
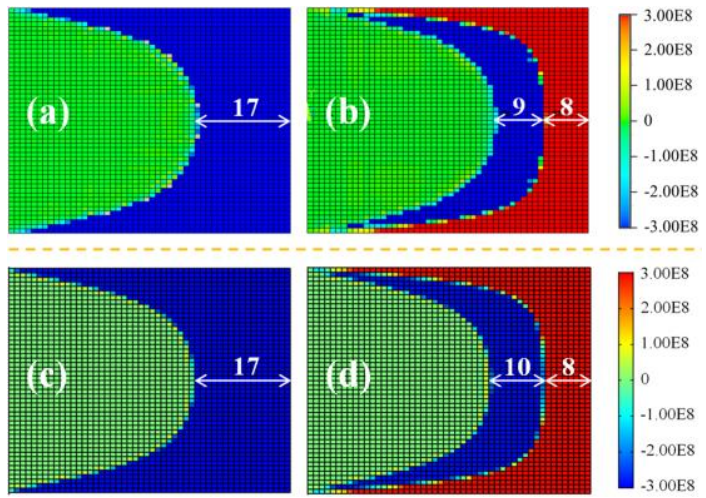

Fig. 2. Magnetization-current density in the bulk HTS (critical state model, $n=200$ ) at (a) $t=500 \mathrm{~s}$ and (b) $t=1000 \mathrm{~s}$ from the ANSYS-IAM. Magnetization-current density in the bulk HTS at (c) $t=500 \mathrm{~s}$ and (d) $t=1000 \mathrm{~s}$ from the COMSOL $\boldsymbol{H}$-formulation.
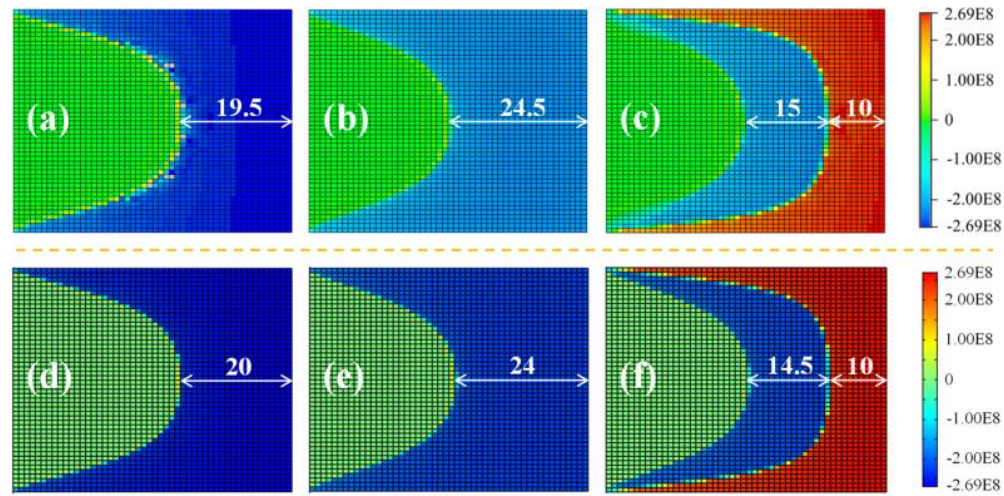

Fig. 3. Magnetization-current density in the bulk HTS (flux creep model, $n=20$ ) at (a) $t$ $=500 \mathrm{~s}$, (b) $t=1000 \mathrm{~s}$ and (c) $t=1500 \mathrm{~s}$ from the ANSYS-IAM. Magnetization-current density in the bulk HTS at (d) $t=500 \mathrm{~s}$, (e) $t=1000 \mathrm{~s}$ and (f) $t=1500 \mathrm{~s}$ from the COMSOL $\boldsymbol{H}$-formulation. where $E_{\mathrm{c}}$ refers to the critical electrical field (usually $10^{-4} \mathrm{~V} / \mathrm{m}$ ) and $n$ indicates the steepness of the transition between the superconducting and the normal state.

Figure 1(a) shows the 2D axis-symmetric half finite-element analysis (FEA) model created in ANSYS 18.1 Academic for computing the magnetization-current in a disk-shaped bulk HTS ( $\Phi 25 \mathrm{~mm}, 10 \mathrm{~mm}$ thick) [21]. The critical current density $J_{\mathrm{c}}$ of the bulk HTS is set to $3 \times 10^{8} \mathrm{~A} / \mathrm{m}^{2}$. The flux-normal boundary (default in ANSYS) is applied to the bottom line and the flux-parallel boundary $\left(A_{z}=0\right)$ is applied to the two outer air-lines. The background magnetic-flux density for magnetizing the bulk HTS is provided by applying source current density to the solenoid. For the critical state model, it rises linearly from zero to $1 \mathrm{~T}$ in $500 \mathrm{~s}$ and then drops to zero in another $500 \mathrm{~s}$, as shown in Fig. 1(b). For the flux creep model, it rises linearly from zero to $1 \mathrm{~T}$ in $500 \mathrm{~s}$ and then holds at $1 \mathrm{~T}$ for $500 \mathrm{~s}$ and finally drops to zero in $500 \mathrm{~s}$, as shown in Fig. 1(c). No field-hold is included in the ZFC magnetization in Fig. 1(b) because the magnetization results obeying the critical state model are time-independent.

\section{THE CRITICAL STATE MODEL}

For modelling the critical state, the initial resistivity $\rho_{0}$ of the bulk HTS is set to a constant value of $10^{-16} \Omega \mathrm{m}$; the 8-nodes 2D element Plane233 with settable degrees of freedom (DOFs) is selected for the electromagnetic analysis; element-type-1 (ET-1, Plane 233, DOF $=A_{Z}$ ) is created in the non-HTS subdomain for computing the magnetic field; element-type-2 (ET-2, Plane 233, DOF $=A_{Z}+V$ ) is created in the HTS subdomain for computing both the magnetic field and the eddy current.

The numerical modelling of the field-ascendant (the first $500 \mathrm{~s}, t_{1}$ ) magnetization follows the iterative algorithm shown in [20]. After each iteration step (200 steps in total, $N_{1}$ ), we obtain the magnetization-current density $\left(\boldsymbol{J}_{\mathrm{T}}\right)$ in each HTS-element and upgrade it with $J_{\mathrm{c}} \cdot \boldsymbol{J}_{\mathrm{T}} / \boldsymbol{J}_{\mathrm{T}} \mid$ if the HTS-element is over-trapped $\left(\left|\boldsymbol{J}_{\mathrm{T}}\right| \geqslant J_{\mathrm{c}}\right)$. This is realized by modifying its element-type to ET-1 and using the "BFE" command. Fig. 2(a) shows the magnetization-current in the bulk HTS after the background magnetic-flux density rises to $1 \mathrm{~T}$ at " $t=500 \mathrm{~s}$ ". All penetrated HTS-elements hold a constant current density of $-3 \times 10^{8} \mathrm{~A} / \mathrm{m}^{2}$. The penetration-depth in the mid-plane of the bulk HTS is 17 -elements ( $17 \times 0.25 \mathrm{~mm})$, showing excellent agreement with the $\boldsymbol{H}$-formulation method ( $n$-value is set to 200 to approach the critical state model) in Fig. 2(c).

The element-type of the penetrated HTS-elements has been modified to ET-1 (inactive eddy-current solver) at " $t=$ $500 \mathrm{~s}$ ". It is therefore essential to reset the element-type of the penetrated HTS-elements to ET-2 to continue modelling the field-descendant magnetization. A viable solution to retain the critical state magnetization-current as much as possible is to 


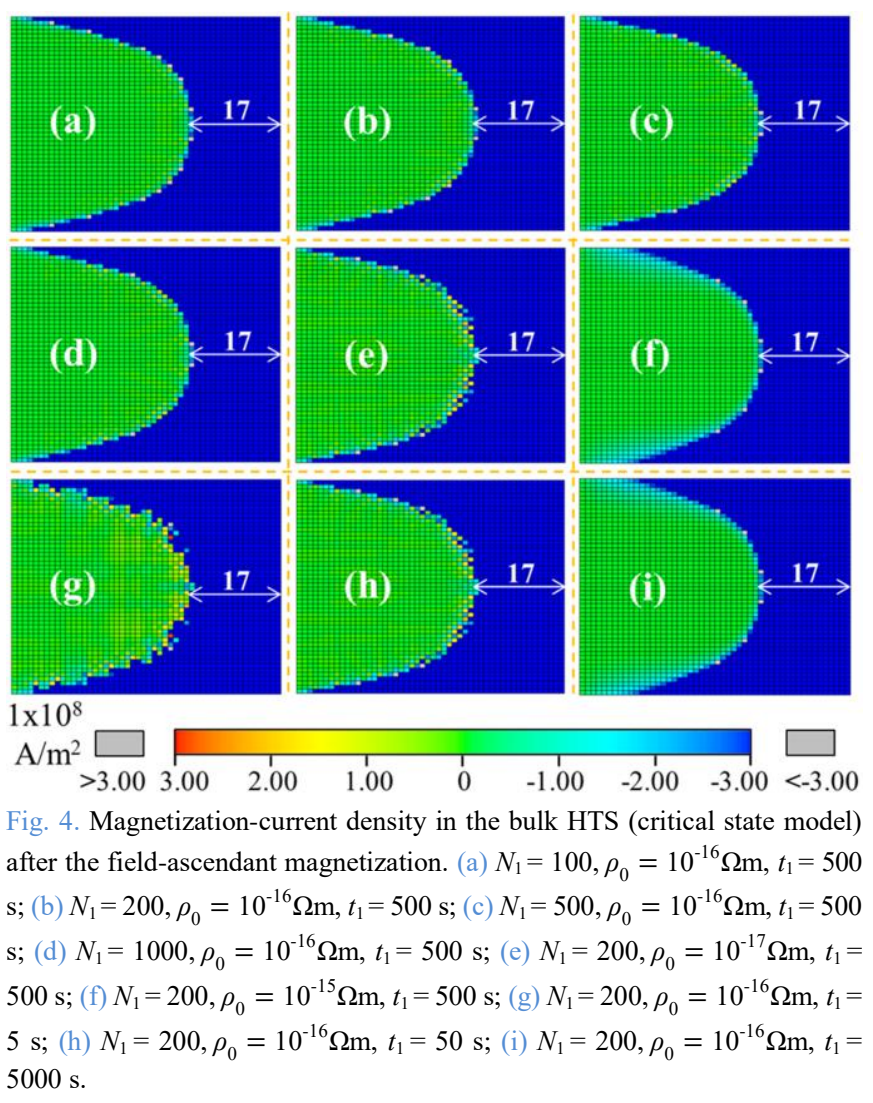

apply voltages $V_{j}$ to the nodes in the penetrated HTS-elements

$$
V_{j}=-2 \pi r_{j} \rho_{0} J_{\mathrm{c}}
$$

where $r_{j}$ refers to the radius of node- $j$ (distance between node- $j$ and the central axis). This setting boundary can minimize the $J_{\mathrm{T}}$-decay in the inner HTS-elements but fortunately will not affect the demagnetization of the outer layer. After another 200 iteration steps $\left(N_{2}=200, t_{2}=500 \mathrm{~s}\right)$ we can obtain the magnetization results after the field-descendant at " $t=1000 \mathrm{~s}$ ". Indeed, the outer HTS-elements trap a constant $\boldsymbol{J}_{\mathrm{T}}$ of $3 \times 10^{8}$ $\mathrm{A} / \mathrm{m}^{2}$ and the inner HTS-elements trap the $\boldsymbol{J}_{\mathrm{T}}$ which is slightly higher than $-3 \times 10^{8} \mathrm{~A} / \mathrm{m}^{2}$. The viable solution to obtain ideal critical state results is to introduce an additional iteration step by using the "BFE" command to force the $\boldsymbol{J}_{\mathrm{T}}$ in the inner HTS-elements to $-3 \times 10^{8} \mathrm{~A} / \mathrm{m}^{2}$. This will result in slight changes of $\boldsymbol{J}_{\mathrm{T}}$ in the neighboring HTS-elements but the numerical modelling of this iteration step obeys to the Maxwell equations. In Fig. 2(b) it can be observed that the penetration depth at " $t=$ $1000 \mathrm{~s}$ " is 8-elements in the outer-layer and 9-elements in the inner-layer. In Figure 2(d) the bulk HTS traps $\sim 2.96 \times 10^{8} \mathrm{~A} / \mathrm{m}^{2}$ in the outer-layer and $\sim-2.90 \times 10^{8} \mathrm{~A} / \mathrm{m}^{2}$ in the inner-layer at " $t=$ $1000 \mathrm{~s}$ ". The trapped $\left|\boldsymbol{J}_{\mathrm{T}}\right|$ is slightly lower than $J_{\mathrm{c}}$ because the flux creep effect cannot be eliminated entirely even if $n$ is set to 200 in the $\boldsymbol{H}$-formulation method. This explains why the penetration-depth of the inner-layer is slightly larger in comparison to the result from the iterative algorithm method.

\section{THE FLUX CREEP MODEL}

To save computation time, the 4-nodes 2D element Plane 13 $\left(\mathrm{DOF}=A_{\mathrm{z}}\right)$, capable of transient electromagnetic analysis, is selected for efficient magnetization simulation of the bulk HTS determined by the flux creep model. The ZFC magnetization-process follows the curve in Fig. 1(c).

The iteration process for flux creep modelling does not require freezing any saturated eddy-currents but will update the resistivity-value of each HTS-element as follows

$$
\rho_{m}^{i+1}=k \rho_{m}^{i}+(1-k) \cdot \max \left\{\rho_{0} \frac{E_{\mathrm{c}}}{J_{\mathrm{c}}} \cdot\left(\frac{\left|\boldsymbol{J}_{\mathrm{T} m}^{i}\right|}{J_{\mathrm{c}}}\right)^{n-1}\right\}
$$

where $\rho_{m}^{i}$ and $\rho_{m}^{i+1}$ refer to the resistivity of element- $m$ at the $i^{\text {th }}$ and the $(i+1)^{\text {th }}$ iteration step, respectively; $\boldsymbol{J}_{\mathrm{T} m}^{i}$ is the obtained magnetization-current density of element $m$ after the $i^{\text {th }}$ iteration step; $k$ is the reservation coefficient for retaining the old resistivity-value and the coefficient " $1-k$ " is for adding the new resistivity-value from the $\boldsymbol{E}-\boldsymbol{J}$ power law.

The electrical-field $\left(\rho \cdot \boldsymbol{J}_{\mathrm{T}}\right)$ in each HTS-element has a continuous value during the iterative electromagnetic analysis. This suggests that any updates of each resistivity-value will result in the associated change of the magnetization-current. Hence, a plenty of iteration steps and a slight change of the resistivity-value of each HTS-element (by defining a large reservation coefficient- $k$ ) after each iteration step are highly appreciated for a smooth simulation of the ZFC magnetization in Fig. 1(c). We should note here that the computation speed slows down as the rise of iteration steps. For example, it can take $1.5 \mathrm{~s}$ for step-1, $4.0 \mathrm{~s}$ for step-200, $6.7 \mathrm{~s}$ for step- $400,10.0 \mathrm{~s}$ for step-600, $12.0 \mathrm{~s}$ for step- $800 \mathrm{~s}$, and $15.0 \mathrm{~s}$ for step-999. ANSYS has a default setting number of 999 for the maximum restart-files (.Rnnn) which strongly recommend users not to define too many restart-steps as it will burden the FEA model and slow down the computation speed.

Figure 3 (a-c) shows the magnetization-current in the bulk HTS at different time-steps from using the ANSYS-IAM. The peak $\left|\boldsymbol{J}_{\mathrm{T}}\right|$ is $2.69 \times 10^{8} \mathrm{~A} / \mathrm{m}^{2}$ and the penetration-depth in the mid-plane is 19.5 -elements after the field-ascendant $(t=500 \mathrm{~s})$; the peak $\left|\boldsymbol{J}_{\mathrm{T}}\right|$ decays to $2.21 \times 10^{8} \mathrm{~A} / \mathrm{m}^{2}$ and the penetration-depth rises to 24.5-elements after the field-hold $(t=1000 \mathrm{~s})$; the demagnetization-layer (red color) rises to 10 -elements and the total penetration-depth rises to 25-elements after the field-descendant $(t=1500 \mathrm{~s})$. In the whole simulation, the $n$-value is set to 20 ; the initial resistivity $\rho_{0}$ is set to $10^{-17} \Omega \mathrm{m}$; the reservation coefficient $k$ is set to 0.99 for the field-ascendant/descendant and 0.9 for the field-hold. The magnetization-current during the field-ascendant/hold, as shown in Fig. 3(a-b), is computed within 900 iteration steps; the magnetization-current after the field-descendant, as shown in Fig. 3(c), is computed within 999 iteration steps (minimized computation time, 700 for the field-ascendant/hold, 299 for the field-descendant). In conclusion, the simulation results of the bulk HTS determined by the flux creep model show excellent agreement with the $\boldsymbol{H}$-formulation method at different time-stages, as shown in Fig. 3(d-f). 
Table I. Magnetization results of the bulk HTS determined by the flux creep model

\begin{tabular}{|lllll|}
\hline Fixed parameters & Altering parameter & $\begin{array}{l}\text { Penetration depth } \\
\text { (-elements) }\end{array}$ & $\boldsymbol{J}_{\mathrm{T}}$-oscillation effect & $\boldsymbol{J}_{\mathrm{T}}$-decay effect \\
\hline$\rho_{0}=10^{-17} \Omega \mathrm{m}, t_{1}=500 \mathrm{~s}, k=0.99$ & $N_{1}=100$ & 18 & Strong & No \\
& $N_{1}=200$ & 19 & Slight & No \\
& $N_{1}=500$ & 20 & No & No \\
& $N_{1}=1000$ & 20 & No & No \\
$N_{1}=500, t_{1}=500 \mathrm{~s}, k=0.99$ & $\rho_{0}=10^{-16} \Omega \mathrm{m}$ & 20 & No & No \\
& $\rho_{0}=10^{-15} \Omega \mathrm{m}$ & 20 & No & Slight \\
$N_{1}=500, \rho_{0}=10^{-17} \Omega \mathrm{m}, k=0.99$ & $t_{1}=5 \mathrm{~s}$ & 17 & Strong & No \\
& $t_{1}=50 \mathrm{~s}$ & 19 & Strong & No \\
& $t_{1}=5000 \mathrm{~s}$ & 23 & No & Strong \\
$N_{1}=500, \rho_{0}=10^{-17} \Omega \mathrm{m}, t_{1}=500 \mathrm{~s}$ & $k=0.9$ & 21 & Slight & Slight \\
& $k=0.8$ & 23 & Strong & Strong \\
& $k=0.7$ & 25 & Strong & Strong \\
\hline
\end{tabular}

(a)

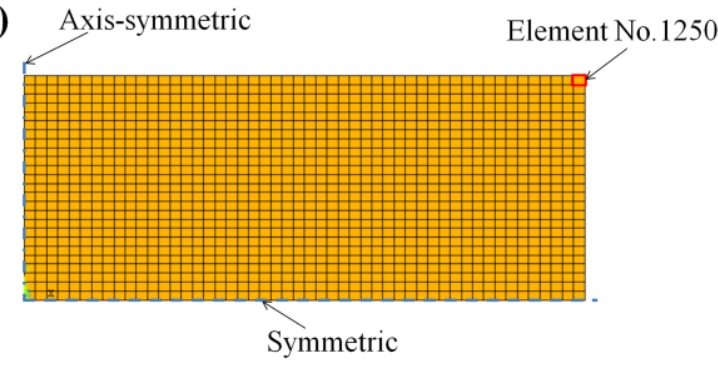

(b)

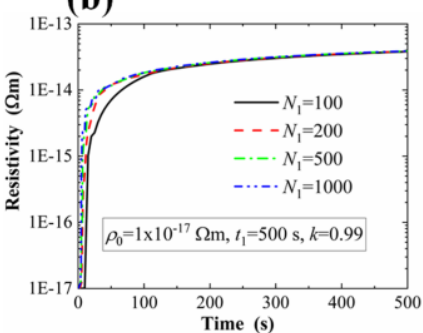

(d)
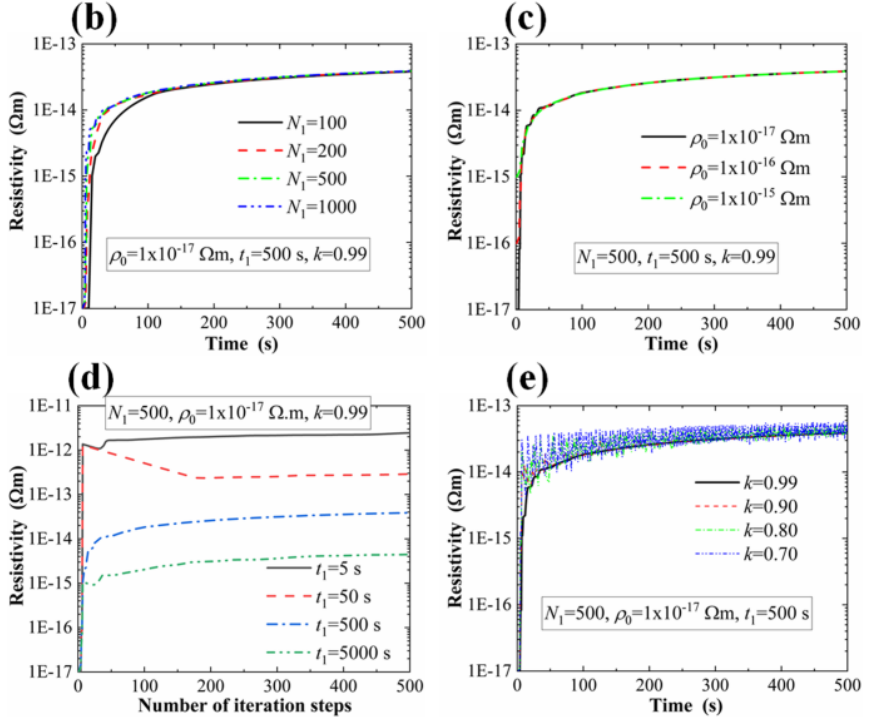

(e)

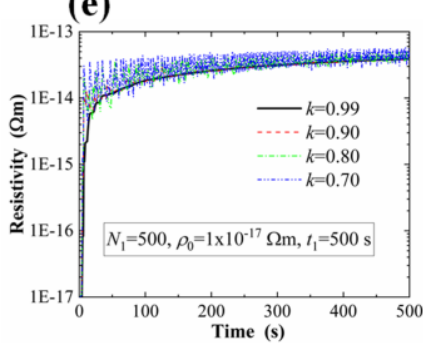

Fig. 5. Resistivity evolution of (a) Element-1250 during the background magnetic-flux density rises linearly from zero to $1 \mathrm{~T}$ (flux creep model, $n=$ 20) when we define varied (b) iteration steps- $N_{1}$; (c) initial resistivity- $\rho_{0}$; (d) ramping time- $t_{1}$; and (e) reservation coefficient $k$.

\section{COMPUTATION ACCURACY}

To better understand the mechanism behind the iterative algorithm method, we re-computed the field-ascendant magnetization for both the critical state model and the flux creep model after changing the setting parameters (iteration steps- $N_{1}$, initial resistivity $\rho_{0}$, ramping time- $t_{1}$, and reservation coefficient- $k$ ).

Fig. 4 compared the magnetization-current in the bulk HTS determined by the critical state model. In Fig. 4(a-d) it can be observed that setting the iteration steps- $N_{1}$ to 100 or larger could obtain similar magnetization results. In Fig. 4(e) and $4(\mathrm{~g}-\mathrm{h})$ it can be observed that there are a few abnormal HTS-elements nearby the penetration-boundary when a low initial resistivity- $\rho_{0}$ or a short ramping time- $t_{1}$ is defined. In Fig. 4(f) and 4(i) it can be observed that there is a slight $\boldsymbol{J}_{\mathrm{T}}$-decay nearby the penetration-boundary when a large $\rho_{0}$ or a large $t_{1}$ is defined.

Table I compares the magnetization results of the bulk HTS determined by the flux creep model. The conclusions are as follows

a) Good magnetization results could be obtained when we define a large amount of iteration steps $\left(N_{1} \geqslant 500\right)$;

b) A slight $\boldsymbol{J}_{\mathrm{T}}$-decay, similar to Fig. 4(f), could occur nearby the penetration boundary when we define a large $\rho_{0}$ (e.g., $\left.10^{-15} \Omega \mathrm{m}\right)$;

c) A short ramping time- $t_{1}$ (e.g., $5 \mathrm{~s}$ and $50 \mathrm{~s}$ ) could bring strong $\boldsymbol{J}_{\mathrm{T}}$-oscillation effects ( $\boldsymbol{J}_{\mathrm{T}}$ jumps up and down, unstable) and result in a small penetration-depth, however, a large $t_{1}$ (e.g., $5000 \mathrm{~s}$ ) could cause strong $J_{\mathrm{T}}$-decay effects and result in a large penetration-depth;

d) A small reservation coefficient- $k$ (e.g., 0.8 and 0.7) could cause strong $\boldsymbol{J}_{\mathrm{T}}$-oscillation and $\boldsymbol{J}_{\mathrm{T}}$-decay, meanwhile, resulting in a "larger" penetration depth.

For better understanding of the iterative algorithm and the computation process of the flux creep model we studied the resistivity-evolution of element-1250 in Fig. 5(a). In Fig. 5(b) it can be observed that the resistivity of element-1250 becomes "dynamic stable" at an earlier time-stage when a larger $N_{1}$ is defined. In Fig. 5(c) it can be observed that the resistivity-curves overlap well for varied $\rho_{0}$ cases. In Fig. $5(\mathrm{~d})$ it can be observed that the "stabilized" resistivity of 
Table II. Comparison of the computation-time based on the flux creep model $(n=20)$

\begin{tabular}{lll}
\hline Method & Field-ascendant-only & Whole ZFC cycle \\
\hline $\boldsymbol{H}$-formulation & $4 \mathrm{~min}$ & $6 \mathrm{~min}$ \\
IAM & $27 \mathrm{~min}$ (500-steps) & $87 \mathrm{~min}$ (999-steps) \\
\hline
\end{tabular}

Table III. Comparison of the computation-time based on the critical state model $(n=200)$

\begin{tabular}{lll}
\hline Method & Field-ascendant-only & Whole ZFC cycle \\
\hline $\boldsymbol{H}$-formulation & $11 \mathrm{~min}$ & $16 \mathrm{~min}$ \\
IAM & $3 \mathrm{~min}(100$-steps $)$ & $13 \mathrm{~min}$ (300-steps) \\
\hline
\end{tabular}

element-1250 is affected by $t_{1}$, exhibiting strong over-trapping effects when a small $t_{1}$ (e.g., $5 \mathrm{~s}$ and $50 \mathrm{~s}$ ) is defined. In Fig. 5(e) it can be observed that there are quite strong $\boldsymbol{J}_{\mathrm{T}}$-oscillation (resistivity-oscillation) effects in element-1250 when a small $k$ (e.g., 0.8 and 0.7 ) is defined.

\section{COMPUTATION TIME}

The above numerical simulations are conducted on a HP-Z8-G4 workstation which uses Intel(R) Xeon(R) Gold $6128 \mathrm{CPU} @ 3.40 \mathrm{GHz}$ and $3.39 \mathrm{GHz}$ (two processors, each one has 6 cores and 12 threads). The FEA models created in ANSYS-IAM and COMSOL $\boldsymbol{H}$-formulation have the same swept-meshing size in the HTS subdomain but random free-meshing size in the non-HTS subdomain (7398 elements in total in ANSYS model and 7376 elements in total in COMSOL model).

Table II and III summarizes the minimum computation-time for modelling the magnetization of the field-ascendant-only and the whole ZFC cycle with the ANSYS-IAM and the COMSOL $\boldsymbol{H}$-formulation. It can be observed that the ANSYS-IAM shows significantly worse performance in flux creep modelling. This is because a large amount of iteration steps are requested to approach the $\boldsymbol{E}-\boldsymbol{J}$ power law and to obtain smooth simulation results. The FEA model is then burdened unfortunately. ANSY-IAM, however, is competitive in modelling the critical state magnetization-current, especially when we only compute the field-ascendant magnetization which is able to be resolved within 100 iteration steps. The iterative algorithm method has similar advantages with the newly proposed backward computation method (no $\boldsymbol{E}-\boldsymbol{J}$ power law equations, no eddy current solving in non-superconductor subdomain, and multi-frame restart analysis) [19]. But it can provide real-time simulation results at any intermediate steps during the magnetization process.

This technical note reported and discussed the key improvements of the iterative algorithm method in comparison to its previous capability [20]. More examples and details, like adding the ferromagnetic materials into the FEA model or taking into account the Kim-model for the bulk HTS, can be found in the original preprint which is an expanded version of this technical note [22].

\section{CONCLUSION}

We have demonstrated that the ANSYS-IAM can model the magnetization-current in the bulk HTS under a whole ZFC cycle for both the critical state model and the flux creep model. In critical state modelling, a special way to minimize the decay of the inner magnetization-current during the demagnetization stage is to apply nodal voltages to all penetrated HTS-elements. In flux creep modelling, it is essential to define a large amount of iteration steps and a large reservation coefficient to obtain accurate simulation results in spite of burdening the FEA model and slowing down the computation speed. Other influence factors, like the ramping time and the initial resistivity of the bulk HTS, are discussed in detail for the field-ascendant process. In conclusion, the ANSYS-IAM shows excellent performance in modelling the real-time magnetization-current obeying the critical state model but relatively poor performance in modelling the real-time magnetization-current obeying the flux creep model. Nevertheless, it is an important step forward to take into account the widely used $\boldsymbol{E}-\boldsymbol{J}$ power law for the iterative algorithm method. Possibilities of optimizing the multi-frame restart analysis and relieving the burden of the FEA model will be investigated.

\section{ACKNOWLEDGMENTS}

The authors would like to thank Dr. Mark Ainslie from University of Cambridge for his helpful discussions and his help in validating our benchmark simulations.

\section{REFERENCES}

[1] M. Calvi, M. D. Ainslie, A. Dennis, J. H. Durrell, S. Hellmann, C. Kittel, D. A. Moseley, T. Schmidt, Y. Shi, and K. Zhang, "A GdBCO bulk staggered array undulator," Supercond. Sci. Technol., vol. 33, Dec. 2019, Art. no. 014004.

[2] S. Hellmann, M. Calvi, T. Schmidt, and K. Zhang, "Numerical design optimization of short-period HTS staggered array undulators," IEEE Trans. Appl. Supercond., vol. 30, no. 4, Jan. 2020, Art. no. 4100705.

[3] G. D'Auria et al., "Status of the CompactLight design study," 39th Free Electron Laser Conf., vol. 39, pp. 738-41, 2020. https://doi.org/10.18429/JACoW-FEL2019-THP078

[4] L. Brouwer, D. Arbelaez, B. Auchmann, L. Bortot, and E. Stubberud, "User defined elements in ANSYS for 2D multiphysics modeling of superconducting magnets," Supercond. Sci. Technol., vol. 32, Aug. 2019, Art. no. 095011.

[5] F. Grilli, S. Stavrev, Y. L. Floch, M. Costa-Bouzo, E. Vinot, I. Klutsch, G. Meunier, P. Tixador, and B. Dutoit, "Finite-element method modeling of superconductors: from 2-D to 3-D," IEEE Trans. Appl. Supercond., vol. 15, no. 1, pp. 17-25, Mar. 2005

[6] S. Stavrev, F. Grilli, B. Dutoit, N. Nibbio, E. Vinot, I. Klutsch, G. Meunier, P. Tixador, Y. Yang, and E. Martinez, "Comparison of numerical methods for modeling of superconductors," IEEE Trans. Appl. Supercond., vol. 38, no. 2, pp. 849-52, Mar. 2002.

[7] N. Amemiya, S. Murasawa, N. Banno, and K. Miyamoto, "Numerical modelings of superconducting wires for AC loss calculations," Physica C, vol. 310, no. 1-4, pp. 16-29, Dec. 1998.

[8] Y. Wang, M. Zhang, F. Grilli, Z. Zhu, and W. Yuan, "Study of the magnetization loss of $\mathrm{CORC}^{\circledR}$ cables using a 3D T-A formulation," Supercond. Sci. Technol., vol. 32, Jan. 2019, Art. no. 025003.

[9] H. Zhang, M. Zhang, and W. Yuan, "An efficient 3D finite element method model based on the T-A formulation for superconducting coated conductors," Supercond. Sci. Technol., vol. 30, Dec. 2016, Art. no. 024005.

[10] Z. Hong, A. M. Campbell, and T. A. Coombs, "Numerical solution of critical state in superconductivity by finite element software," Supercond. Sci. Technol., vol. 19, pp. 1246-52, Oct. 2006. 
[11] R. Brambilla, F. Grilli, and L. Martini, "Development of an edge-element model for AC loss computation of high-temperature superconductors," Supercond. Sci. Technol., vol. 20, pp. 16-24, Nov. 2006.

[12] M. Zhang and T. A. Coombs, "3D modeling of high- $\mathrm{T}_{\mathrm{c}}$ superconductors by finite element software," Supercond. Sci. Technol., vol. 25, Dec. 2011, Art. no. 015009.

[13] M. D. Ainslie, V. M. Rodriguez-Zermeno, Z. Hong, W. Yuan, T. J. Flack, and T. A. Coombs, "An improved FEM model for computing transport AC loss in coils made of RABiTS YBCO coated conductors for electric machines," Supercond. Sci. Technol., vol. 24, Jan. 2011, Art. no. 045005.

[14] B. Shen, F. Grilli, and T. Coombs, "Review of the AC loss computation for HTS using H formulation," Supercond. Sci. Technol., vol. 33, Feb. 2020, Art. no. 033002.

[15] E. Pardo and M. Kapolka, "3D computation of non-linear eddy currents: Variational method and superconducting cubic bulk," J. Comput. Phys., vol. 344, pp. 339-63, Sep. 2017.

[16] C. Gu and Z. Han, "Calculation of AC losses in HTS tape with FEA program ANSYS," IEEE Trans. Appl. Supercond., vol. 15, no. 2, pp. 2859-62, Jun. 2005.

[17] S. Farinon, G. Iannone, P. Fabbricatore, and U. Gambardella, "2D and 3D numerical modeling of experimental magnetization cycles in disks and spheres," Supercond. Sci. Technol., vol. 27, Sep. 2014, Art. no. 104005.

[18] C. Gu, T. Qu, X. Li, and Z. Han, "AC losses in HTS tapes and devices with transport current solved through the resistivity-adaption algorithm," IEEE Trans. Appl. Supercond., vol. 23, no. 2, Apr. 2013, Art. no. 8201708.

[19] K. Zhang, M. Ainslie, M. Calvi, S. Hellmann, R. Kinjo, and T. Schmidt, "Fast and efficient critical state modelling of field-cooled bulk high-temperature superconductors using a backward computation method," Supercond. Sci. Technol., vol. 33, Oct. 2020, Art. no. 114007.

[20] K. Zhang, S. Hellmann, M. Calvi, and T. Schmidt, "Magnetization simulation of ReBCO tape stack with a large number of layers using the ANSYS A-V-A Formulation," IEEE Trans. Appl. Supercond., vol. 30, no. 4, Jun. 2020, Art. no. 4700805.

[21] M. Ainslie and J. Zou, Benchmark test, HTS modelling workgroup, 2015. http://www.htsmodelling.com/?wpdmpro=b4 results

[22] K. Zhang, S. Hellmann, M. Calvi, T. Schmidt, and L. Brouwer, "Magnetization current simulation of high temperature bulk superconductors using A-V-A formulation and iterative algorithm method: critical state model and flux creep model," arXiv:1908.04640v2, Feb. 2020.

Kai Zhang received the B.E., degree in Mechanical Engineering from Nanjing Tech University, Nanjing, China, in 2011, the M.E. and Ph.D. degrees in "Mechanical Engineering" from University of Chinese Academy of Sciences, Beijing, China, in 2014 and 2018, respectively. His PhD thesis on the R\&D of high field superconducting accelerator dipole magnets was completed at the Institute of high energy physics, Beijing and the Lawrence Berkeley National Laboratory, California. Since 2018 he has been working as a postdoc fellow at the Insertion Device group of the Paul Scherrer Institute, Villigen, Switzerland. His current research interests include the advanced electromagnetic analysis of large scale high-temperature superconductor (HTS) models and study of novel HTS undulator technologies.

Sebastian Hellmann received his German Diploma-degree in "Electrical Engineering" in 2010 and his M.Sc. in "Automation and Energy Systems" in 2012, both from the University of Applied Sciences in Mannheim, Germany. His Ph.D. he obtained at the Karlsruhe Institute of Technology, Kalrsruhe, Germany in 2018 for his work on superconducting transformers. From 2018 he was a Marie Skłodowska-Curie Postdoctoral Fellow in the field of superconducting undulators for compact light sources at the Paul Scherrer Institut in Villigen, Switzerland for two years, until he joined a group for superconducting accelerator magnet development at the same institution in 2020 .

Marco Calvi is an accelerator physicist working since 2009 at the Photon Science Division of the Paul Scherrer Institute (PSI). He received his $\mathrm{PhD}$ in 2004 at the University of Geneva in the Department of Quantum Material Physics under the supervision of Prof. R. Flükiger. Then, he started his career at CERN during the construction of the Large Hadron Collider (LHC) in the domain of high field superconducting magnets. Later, he moved to EPFL for joining the ITER project and the cold cable tests of its superconducting Tokamak. At the beginning of the SwissFEL project, Dr. Calvi was hired by PSI to work on the undulator system of its first hard X-ray beamline, Aramis. Today, after more than 10 years, he is an experienced physicist in the domain of accelerator-based light sources, both synchrotrons and FELs. His favourite research field is High Temperature Superconducting Undulators (HTSU).

Thomas Schmidt studied physics at the University of Dortmund where he did his $\mathrm{PhD}$ with a storage ring free electron laser. In 1998 he joint the Paul Scherrer Institut in 1998 with the start of the SLS project. Together with Gerhard Ingold he was responsible for the Insertion Devices. In collaborations with SPring-8 for hard $\mathrm{x}$-ray in-vacuum undulators to be operated at high harmonics and BESSY II (now HZB) for soft x-ray APPLE II undulators the first set of beamlines has been realized for the start of SLS in 2001. The portfolio was completed with a quasi-period electromagnet undulator for the VUV and a wiggler for highest photon energies demanded by the Materials Science beamline. With the integration of SLS into the organizational structure of PSI, Thomas Schmidt became group leader of the newly established Insertion Device group. In the following years all straight sections have been successive equipped, amongst other with the first fixed-gap APPLE II undulator and one of the first cryogenic in-vacuum undulators. Based on the experience with SLS he developed with his team new concepts for the SwissFEL hard- and soft $\mathrm{x}$-ray undulator lines, namely wedge-based high precision in-vacuum undulators with an automated optimization and soft x-ray undulators in the APPLE X configuration, a full symmetric design with the option of transverse gradients.

Lucas Brouwer, biography not available at the time of publication. 